\title{
Quantum Curve and the First Painlevé Equation
}

\author{
Kohei IWAKI ${ }^{\dagger}$ and Axel SAENZ $\ddagger$ \\ $\dagger$ Graduate School of Mathematics, Nagoya University, Nagoya, 464-8602, Japan \\ E-mail: iwaki@math.nagoya-u.ac.jp \\ $\ddagger$ Department of Mathematics, University of California, Davis, CA 95616-8633, USA \\ E-mail: asaenz@math.ucdavis.edu
}

Received August 04, 2015, in final form January 22, 2016; Published online January 29, 2016

http://dx.doi.org/10.3842/SIGMA.2016.011

\begin{abstract}
We show that the topological recursion for the (semi-classical) spectral curve of the first Painlevé equation $P_{\mathrm{I}}$ gives a WKB solution for the isomonodromy problem for $P_{\mathrm{I}}$. In other words, the isomonodromy system is a quantum curve in the sense of [Dumitrescu O., Mulase M., Lett. Math. Phys. 104 (2014), 635-671, arXiv:1310.6022] and [Dumitrescu O., Mulase M., arXiv:1411.1023].

Key words: quantum curve; first Painlevé equation; topological recursion; isomonodoromic deformation; WKB analysis
\end{abstract}

2010 Mathematics Subject Classification: 34M55; 81T45; 34M60; 34M56

Dedicated to Professor Takahiro Kawai on his seventieth birthday

\section{Introduction}

Painlevé transcendents are remarkable special functions which appear in many areas of mathematics and physics (e.g., [17]). These are solutions of certain nonlinear ordinary differential equations known as Painlevé equations. These equations were discovered by Painlevé and Gambier more than 100 years ago [34], and solutions have the so-called Painlevé property; i.e., any movable singularity must be a pole. One particular property of the Painlevé equations is existence of the Lax pair; that is, each Painlevé equation describes an isomonodromic deformation of a certain meromorphic linear ordinary differential equation [20,21]. The monodromy data of the linear ODEs gives a conserved quantity of the Painlevé transcendents. The RiemannHilbert method, as well as exact WKB analysis are applied to analyze the properties of Painlevé transcendents $[4,17,24,25,36]$.

On the other hand, quantum curves attract both mathematicians and physicists since they are expected to encode the information of many quantum topological invariants, such as GromovWitten invariants, quantum knot invariants etc. These are concieved in physics literature including $[1,2,10,18]$. A quantum curve is an ordinary differential (or difference) equation containing a formal parameter $\hbar$ (which plays the role of the Planck constant), like a Schrödinger equation. The quantum invariants appear in the coefficients of the WKB (Wentzel-Kramers-Brillouin) solution of the quantum curve.

The Eynard-Orantin's topological recursion introduced in [16] is closely related to both of the quantum curves and Painlevé equations (and many other topics). Topological recursion is a recursive algorithm to compute the $1 / N$-expansion of the correlation functions and the partition function of matrix models from its spectral curve, and it is generalized to any algebraic curve which may not come from a matrix model. In this context, quantum curves were first discussed in [6] for the Airy spectral curve, and generalized to spectral curves with various backgrounds (see [11, 12, 13, 18, 29] and the survey article [31]). The spectral curves are recovered as the semi-classical limit $\hbar \rightarrow 0$ of the quantum curves. Moreover, the topological 
recursion is also closely related to integrability $[5,7,19]$ as is the relationship between matrix models and integrable systems [9, 28].

The aim of this paper is to relate quantum curves and the first Painlevé equation with a formal parameter $\hbar$

$$
P_{\mathrm{I}}: \hbar^{2} \frac{d^{2} q}{d t^{2}}=6 q^{2}+t
$$

The (semi-classical) spectral curve for the isomonodormy system associated with $P_{\mathrm{I}}$ is given by

$$
y^{2}=4\left(x-q_{0}\right)^{2}\left(x+2 q_{0}\right),
$$

where $q_{0}=q_{0}(t)$ is an explicit function of $t$. This is a family of algebraic curves in $(x, y)$ space parametrized by $t$. (The curve (1.1) appeared in [16, Section 10.6] as the spectral curve of (3,2)-minimal model.) Our main result claims that, starting from the spectral curve (1.1), its quantization through the Eynard--Orantin's topological recursion (in the sense of [11, 12]) recovers the whole isomonodoromy system for $P_{\mathrm{I}}$.

The precise statement of our main theorem is as follows. Let $W_{g, n}\left(z_{1}, \ldots, z_{n}\right)$ be the EynardOrantin differential of type $(g, n)$ defined from the spectral curve (1.1) (see Section 3.1). These are meromorphic multi-differential forms, and $z_{i}$ 's are copies of a coordinate on the spectral curve (1.1). $W_{g, n}$ 's also depend on $t$ since the spectral curve depends on $t$. Then, our main result states the following.

Theorem 1.1 (Theorem 3.3). The following WKB-type formal series $\psi(x, t, \hbar)$ defined by

$$
\psi(x(z), t, \hbar):=\exp \left(\sum_{g \geq 0, n \geq 1} \hbar^{2 g-2+n} \frac{1}{n !} \frac{1}{2^{n}} \int_{\bar{z}}^{z} \cdots \int_{\bar{z}}^{z} W_{g, n}\left(z_{1}, \ldots, z_{n}\right)\right)
$$

satisfies the isomonodromy system associated with $P_{\mathrm{I}}$. Here $x(z)$ is an explicit rational function of $z$ which appears in the parametrization of the spectral curve (1.1), and $\bar{z}=-z$.

The above theorem tells us that the isomonodoromy system associated with $P_{\mathrm{I}}$ is a quantum curve, and its particular WKB solution is constructed by the topological recursion as (1.2). The main differences between our theorem and previous results on quantum curves are the following:

- Our quantum curve is a restriction of a certain partial differential equation (a holonomic system).

- There are infinitely many $\hbar$-correction terms in the quantum curve, and these correction terms are essentially given by the asymptotic expansion of the solution of $P_{\mathrm{I}}$ for $\hbar \rightarrow 0$.

This paper is organized as follows. In Section 2, we briefly review some known facts about $P_{\mathrm{I}}$ together with an important result on the WKB analysis of isomonodromic systems developed by Kawai-Takei [25, 26]. Our main theorem will be formulated in Section 3 after recalling the notion of topological recursion. We will give a proof of the main results in Section 4.

Remark 1.2. After writing the draft version of this paper, the authors were informed that B. Eynard also has the same result which has not been published yet, but presented in [14]. See also [15, Chapter 5]. 


\section{The first Painlevé equation and isomonodromy system}

Let us consider the first Painlevé equation with a formal parameter $\hbar$ :

$$
P_{\mathrm{I}}: \hbar^{2} \frac{d^{2} q}{d t^{2}}=6 q^{2}+t
$$

The equation $P_{\mathrm{I}}$ is obtained from

$$
\frac{d^{2} \tilde{q}}{d \tilde{t}^{2}}=6 \tilde{q}^{2}+\tilde{t}
$$

via the rescaling $\tilde{t}=\hbar^{-4 / 5} t, \tilde{q}=\hbar^{-2 / 5} q$. We will regard $\hbar$ as a small parameter (i.e., Planck's constant), and investigate a particular formal solution of $P_{\mathrm{I}}$ which has an $\hbar$-expansion.

\subsection{Formal solution of $\boldsymbol{P}_{\mathrm{I}}$}

$P_{\mathrm{I}}$ has the following formal power series solution:

$$
q(t, \hbar)=\sum_{n=0}^{\infty} \hbar^{2 n} q_{2 n}(t)=q_{0}(t)+\hbar^{2} q_{2}(t)+\hbar^{4} q_{4}(t)+\cdots
$$

It contains only even order terms of $\hbar$ since $P_{\mathrm{I}}$ is invariant under $\hbar \mapsto-\hbar$. The leading term $q_{0}=q_{0}(t)$ satisfies

$$
6 q_{0}^{2}+t=0, \quad \text { hence } q_{0}(t)=\sqrt{-t / 6}
$$

and the subleading terms are recursively determined by

$$
q_{2(k+1)}(t)=\frac{1}{12 q_{0}(t)}\left(\frac{d^{2} q_{2 k}}{d t^{2}}(t)-6 \sum_{k_{1}+k_{2}=k+1, k_{i}>0} q_{2 k_{1}}(t) q_{2 k_{2}}(t)\right), \quad k \geq 1 .
$$

As we will see, the coefficients of the formal series appearing in this paper are multivalued functions of $t$ and are defined on the Riemann surface of $q_{0}$. Thus, in what follows, we may use $q_{0}$ instead of $t$ when we express coefficients.

The relation (2.3) implies

$$
q_{2 k}=c_{2 k} q_{0}^{1-5 k}, \quad c_{2 k} \in \mathbb{C} .
$$

It is obvious that the coefficients $q_{2 k}(t)$ have a singularity at $q_{0}=0$ (i.e., $t=0$ ). This special point is called a turning point of $P_{\mathrm{I}}[25$, Definition 2.1] (see also [26, Section 4]). Throughout the paper, we assume the following:

Assumption 2.1. The independent variable $t$ of $P_{\mathrm{I}}$ lies on a domain that doesn't contain the origin.

Remark 2.2. The formal solution (2.1) is called a 0-parameter solution of $P_{\mathrm{I}}$ in [26] since it doesn't contain free parameters. More general formal solutions having one or two free parameters (called 1- or 2-parameter solutions) are constructed in [4] for all Painlevé equations of second order. See also [3] for a construction of general formal solutions of higher order Painlevé equations. 
Remark 2.3. The formal solution (2.1) is in fact a divergent series. However, [23, Theorem 1.1] proved that the formal solution is Borel summable when $q_{0}$ satisfies $q_{0} \neq 0$ and $\arg q_{0} \notin\left\{\frac{2 \ell}{5} \pi \mid \ell \in \mathbb{Z}\right\}$. The exceptional set is called the Stokes curve of $P_{\mathrm{I}}$. (See [25, Definition 2.1] for the notion of Stokes curves of Painlevé equations with a small parameter $\hbar$.) That is, there exists a function which is analytic in $\hbar$ on a sectorial domain with the center at the origin (which is also analytic in $t$ ) such that (2.1) is the asymptotic expansion of the function for $\hbar \rightarrow 0$ in the sector. The analytic function is called the Borel sum of the formal series (2.1), and it gives an analytic solution of $P_{\mathrm{I}}$ (see [8] for Borel summation method). This particular asymptotic solution obtained by the Borel summation method is called the tri-tronquée solution of $P_{\mathrm{I}}$ (see [22]), and the non-linear Stokes phenomena on Stokes curves are analyzed by [17, 24, 36].

\subsection{Isomonodromy system and the $\tau$-function}

It is known that $P_{\mathrm{I}}$ describes the compatibility condition for the following system of linear PDEs (cf. [21, Appendix C]):

$$
\hbar \frac{\partial \Psi}{\partial x}=A \Psi, \quad \hbar \frac{\partial \Psi}{\partial t}=B \Psi
$$

where

$$
\begin{aligned}
& A=\left(\begin{array}{ll}
A_{11} & A_{12} \\
A_{21} & A_{22}
\end{array}\right):=\left(\begin{array}{cc}
p & 4(x-q) \\
x^{2}+q x+q^{2}+\frac{t}{2} & -p
\end{array}\right), \\
& B=\left(\begin{array}{ll}
B_{11} & B_{12} \\
B_{21} & B_{22}
\end{array}\right):=\left(\begin{array}{ll}
0 & 2 \\
\frac{x}{2}+q & 0
\end{array}\right) .
\end{aligned}
$$

The compatibility condition

$$
\hbar \frac{\partial A}{\partial t}-\hbar \frac{\partial B}{\partial x}+[A, B]=0
$$

is equivalent to the following Hamiltonian system

$$
\hbar \frac{d q}{d t}=\frac{\partial H}{\partial p}, \quad \hbar \frac{d p}{d t}=-\frac{\partial H}{\partial q},
$$

where the (time-dependent) Hamiltonian is given by

$$
H=H(q, p, t):=\frac{1}{2} p^{2}-2 q^{3}-t q .
$$

We can easily check that (2.5) and $P_{\mathrm{I}}$ are equivalent. The above system of linear ODEs is called the isomonodromy system associated with $P_{\mathrm{I}}$ (see $[20,21]$ ).

Let $(q, p)=(q(t, \hbar), p(t, \hbar))$ be a formal power series solution of the Hamiltonian system (2.5); that is, $q(t, \hbar)$ is the formal solution $(2.1)$ of $P_{\mathrm{I}}$, and

$$
p(t, \hbar)=\hbar \frac{d q(t, \hbar)}{d t}=\sum_{n=0}^{\infty} \hbar^{2 n+1} p_{2 n+1}(t) .
$$

The corresponding Hamiltonian function is denoted by

$$
\sigma(t, \hbar):=H(q(t, \hbar), p(t, \hbar), t) .
$$

We can check that (2.6) is invariant under $\hbar \mapsto-\hbar$, and hence it has the following expansion:

$$
\sigma(t, \hbar)=\sum_{n=0}^{\infty} \hbar^{2 n} \sigma_{2 n}(t) .
$$


Definition 2.4 ([20,32]). The $\tau$-function (corresponding to the formal solution $(2.1)$ ) of $P_{\mathrm{I}}$ is defined by

$$
\hbar^{2} \frac{d}{d t} \log \tau(t, \hbar)=\sigma(t, \hbar)
$$

up to constant.

The $\tau$-function can also be defined in terms of a solution of (2.4) [20] (see also Appendix A). The expansion (2.7) implies that the $\tau$-function (2.8) has an expansion of the form

$$
\log \tau(t, \hbar)=\sum_{g=0}^{\infty} \hbar^{2 g-2} \tau_{2 g}(t)
$$

\subsection{Spectral curve}

In what follows, we assume that the formal solution $(q(t, \hbar), p(t, \hbar))$ of $(2.5)$ constructed above is substituted into the coefficients of the isomonodromy system (2.4). Then, the coefficients of the isomonodromy system has the following $\hbar$-expansions:

$$
\begin{aligned}
& A=A_{0}(x, t)+\hbar A_{1}(x, t)+\hbar^{2} A_{2}(x, t)+\cdots, \\
& B=B_{0}(x, t)+\hbar B_{1}(x, t)+\hbar^{2} B_{2}(x, t)+\cdots,
\end{aligned}
$$

whose top terms are given by

$$
A_{0}(x, t)=\left(\begin{array}{cc}
0 & 4\left(x-q_{0}\right) \\
x^{2}+q_{0} x+q_{0}^{2}+\frac{t}{2} & 0
\end{array}\right), \quad B_{0}(x, t)=\left(\begin{array}{cc}
0 & 2 \\
\frac{x}{2}+q_{0} & 0
\end{array}\right) .
$$

Observe that, since $q_{0}$ satisfies (2.2), the algebraic curve defined by

$$
\operatorname{det}\left(y-A_{0}(x, t)\right)=y^{2}-4\left(x-q_{0}\right)^{2}\left(x+2 q_{0}\right)=0
$$

has genus 0 . Actually, this gives a family of algebraic curves in $\mathbb{C}_{(x, y)}^{2}$ parametrized by $t$. Since we have assumed that $t \neq 0, x=q_{0}$ and $x=-2 q_{0}$ are distinct.

Definition 2.5. We call the algebraic curve (2.9) the semi-classical spectral curve, or the spectral curve of (the first equation of) the isomonodromy system (2.4).

Remark 2.6. It is shown in [25, Proposition 1.3] that, for all (second order) Painlevé equations with a formal parameter $\hbar$, the semi-classical spectral curves corresponding to the same type of formal power series solution as (2.1) have genus 0 .

Remark 2.7. Since we are taking the semi-classical limit (i.e., top term in $\hbar$-expansion), our spectral curve (2.9) is different from usual spectral curves for isomonodromic deformation equations discussed, e.g., in [33, 35]. The spectral curves in the above papers have higher genus. Recently, Nakamura [30] investigates the geometry of genus 2 spectral curves which appear in an autonomous limit of the 4th order Painlevé equations, and use them to classify the Painlevé equations. See [27] for the list of 4th order Painlevé equations. 


\subsection{WKB analysis of isomonodromy system in scalar form}

Denote the unknown vector function of $(2.4)$ by $\Psi={ }^{t}\left(\psi_{1}, \psi_{2}\right)$. Then, $\psi=\psi_{1}$ satisfies the following scalar version of isomonodromy system

$$
\begin{aligned}
& \left(\left(\hbar \frac{\partial}{\partial x}\right)^{2}+f\left(\hbar \frac{\partial}{\partial x}\right)+g\right) \psi=0, \\
& \hbar \frac{\partial \psi}{\partial t}=\frac{1}{2(x-q)}\left(\hbar \frac{\partial \psi}{\partial x}-p \psi\right),
\end{aligned}
$$

where

$$
\begin{aligned}
f & =f(x, t, \hbar):=-\operatorname{tr} A-\hbar \frac{\partial}{\partial x} \log A_{12}=-\hbar \frac{1}{x-q} \\
g & =g(x, t, \hbar):=\operatorname{det} A-\hbar \frac{\partial A_{11}}{\partial x}+\hbar A_{11} \frac{\partial}{\partial x} \log A_{12} \\
& =-\left(4 x^{3}+2 t x+p^{2}-4 q^{3}-2 t q\right)+\hbar \frac{p}{x-q} .
\end{aligned}
$$

The coefficients of $f$ and $g$ have an $\hbar$-expansion since $q$ and $p$ are contained in them

$$
\begin{aligned}
& f=-\hbar \frac{1}{x-q_{0}}+\hbar^{3} \frac{1}{1728 q_{0}^{4}\left(x-q_{0}\right)^{2}}+\hbar^{5} \frac{49 x-51 q_{0}}{5971968 q_{0}^{9}\left(x-q_{0}\right)^{3}}+\cdots, \\
& g=-4\left(x-q_{0}\right)^{2}\left(x+2 q_{0}\right)-\hbar^{2} \frac{x+11 q_{0}}{144 q_{0}^{2}\left(x-q_{0}\right)}-\hbar^{4} \frac{7 x^{2}+34 q_{0} x-53 q_{0}^{2}}{248832 q_{0}^{7}\left(x-q_{0}\right)^{2}}+\cdots .
\end{aligned}
$$

The top term of $g$ appears in the defining equation of the spectral curve (2.9), and its zeros are called turning points of the first equation of (2.10) in the WKB analysis. In particular, under the assumption $t \neq 0$, there is

- a simple turning point at $x=-2 q_{0}$ which is a branch point of the spectral curve $(2.9)$, and

- a double turning point at $x=q_{0}$ which is a singular point of the spectral curve (2.9).

Consider the Riccati equation

$$
\hbar^{2}\left(P^{2}+\frac{\partial P}{\partial x}\right)+f \hbar P+g=0 .
$$

This is equivalent to the first equation in (2.10) by

$$
\psi=\exp \left(\int^{x} P d x\right), \quad \text { i.e., } \quad P=\frac{1}{\psi} \frac{\partial \psi}{\partial x} .
$$

Let

$$
P^{( \pm)}(x, t, \hbar)=\sum_{m=0}^{\infty} \hbar^{m-1} P_{m}^{( \pm)}(x, t)
$$

be the formal solutions of (2.13) with the top term

$$
P_{0}^{( \pm)}(x, t)= \pm 2\left(x-q_{0}\right) \sqrt{x+2 q_{0}} .
$$


The coefficients $P_{m}^{( \pm)}(x, t)$ are recursively determined by

$$
2 P_{0}^{( \pm)} P_{m+1}^{( \pm)}+\sum_{\substack{a+b=m+1 \\ a, b \geq 1}} P_{a}^{( \pm)}\left(P_{b}^{( \pm)}+f_{b}\right)+\frac{\partial P_{m}^{( \pm)}}{\partial x}+g_{m+1}=0 \quad \text { for } \quad m \geq 0
$$

where $f_{a}$ and $g_{a}$ are the coefficient of $\hbar^{a}$ in $f$ and $g$, respectively. Explicit forms of the first few terms are given by

$$
\begin{aligned}
& P_{1}^{( \pm)}=-\frac{1}{4\left(x+2 q_{0}\right)}, \quad P_{2}^{( \pm)}= \pm \frac{x+17 q_{0}}{576 q_{0}^{2}\left(x+2 q_{0}\right)^{5 / 2}}, \quad P_{3}^{( \pm)}=-\frac{2 x^{2}+20 q_{0} x+77 q_{0}^{2}}{6912 q_{0}^{4}\left(x+2 q_{0}\right)^{4}} \\
& P_{4}^{( \pm)}= \pm \frac{28 x^{4}+500 q_{0} x^{3}+3684 q_{0}^{2} x^{2}+14273 q_{0}^{3} x+27307 q_{0}^{4}}{3981312 q_{0}^{7}\left(x+2 q_{0}\right)^{11 / 2}}
\end{aligned}
$$

It is obvious from $(2.14)$ that $P_{m}^{( \pm)}(x, t)$ are holomorphic except at the turning points and $x=\infty$ (and multivalued for even $m$ ). It also follows from the recursion relation (2.14) that

$$
P^{( \pm)}(x, t, \hbar)= \pm\left(\frac{2}{\hbar} x^{3 / 2}+\frac{t}{2 \hbar} x^{-1 / 2} \mp \frac{1}{4} x^{-1}+\frac{\sigma(t, \hbar)}{2 \hbar} x^{-3 / 2}+O\left(x^{-2}\right)\right)
$$

holds when $x \rightarrow \infty$.

Remark 2.8. We can check that $P_{m}^{( \pm)}(x, t)$ 's have the following asymptotic expansion for $x \rightarrow \infty$

$$
\begin{aligned}
& P_{0}^{( \pm)}(x, t)= \pm\left(2 x^{3 / 2}+\frac{t}{2} x^{-1 / 2}+O\left(x^{-3 / 2}\right)\right), \quad P_{1}^{( \pm)}(x, t)=-\frac{1}{4} x^{-1}+O\left(x^{-3 / 2}\right), \\
& P_{m}^{( \pm)}(x, t)=O\left(x^{-3 / 2}\right) \quad \text { for } \quad m \geq 2,
\end{aligned}
$$

and we have (2.15) after summing up $\hbar^{m-1} P_{m}^{( \pm)}(x, t)$. Once you know that $P^{( \pm)}(x, t, \hbar)$ has an asymptotic expansion in this sense, subleading terms in (2.15) can be computed from the Riccati equation (2.13).

Define

$$
\begin{aligned}
& P_{\text {odd }}(x, t, \hbar):=\frac{1}{2}\left(P^{(+)}(x, t, \hbar)-P^{(-)}(x, t, \hbar)\right), \\
& P_{\text {even }}(x, t, \hbar):=\frac{1}{2}\left(P^{(+)}(x, t, \hbar)+P^{(-)}(x, t, \hbar)\right) .
\end{aligned}
$$

It is easy to check that (cf. [26, Section 2])

$$
P_{\text {even }}(x, t, \hbar)=-\frac{1}{2} \frac{\partial}{\partial x} \log \frac{\hbar P_{\text {odd }}(x, t, \hbar)}{2(x-q(t, \hbar))}
$$

and

$$
P_{\text {odd }}(\sigma(x), t, \hbar)=-P_{\text {odd }}(x, t, \hbar)
$$

hold. Here $x$ is regarded as a coordinate on the spectral curve, and $\sigma$ is the covering involution for the spectral curve: $P^{(+)}(\sigma(x), t)=-P^{(-)}(x, t)$.

Since

$$
\frac{\hbar P_{\text {odd }}(x, t, \hbar)}{2(x-q(t, \hbar))}=\sqrt{x+2 q_{0}}(1+O(\hbar)),
$$




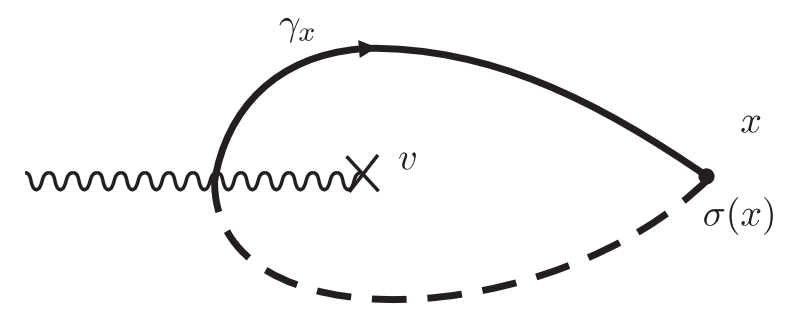

Figure 1. For a given $x$, the path $\gamma_{x}$ starts from the point $\sigma(x)$ and ends at $x$. The wiggly lines designate a branch cut, and the solid (resp. dotted) part represents a part of path on the first (resp. the second) sheet of the spectral curve.

the right hand-side of (2.16) is the derivative of the formal power series

$$
-\frac{1}{2} \log \frac{\hbar P_{\text {odd }}(x, t, \hbar)}{2(x-q(t, \hbar))}=-\frac{1}{4} \log \left(x+2 q_{0}\right)+O(\hbar) .
$$

Thus the ambiguity of the branch of the logarithm only appears in the top term, but we care about the ambiguity since it doesn't matter in our computation.

The following theorem was applied in the transformation theory of Painlevé equations in [25]. We will use the fact in the proof of our main theorem.

Theorem 2.9 (cf. [25, Proposition 1.2 and Theorem 1.1]).

(i) The formal series $P^{( \pm)}(x, t, \hbar)$ satisfies

$$
\hbar \frac{\partial}{\partial t} P^{( \pm)}(x, t, \hbar)=\frac{\partial}{\partial x}\left(\frac{\hbar P^{( \pm)}(x, t, \hbar)-p(t, \hbar)}{2(x-q(t, \hbar))}\right) .
$$

In particular, $P_{\text {odd }}(x, t, \hbar)$ satisfies

$$
\frac{\partial}{\partial t} P_{\text {odd }}(x, t, \hbar)=\frac{\partial}{\partial x}\left(\frac{P_{\text {odd }}(x, t, \hbar)}{2(x-q(t, \hbar))}\right) .
$$

(ii) All coefficients of $P^{( \pm)}(x, t, \hbar)$ are holomorphic except at the simple turning point $x=-2 q_{0}$ and $x=\infty$. In particular, they are holomorphic at the double turning point $x=q_{0}$.

(iii) The formal series

$$
\begin{aligned}
\psi_{ \pm}(x, t, \hbar) & :=\exp \left( \pm \int_{v}^{x} P_{\text {odd }}\left(x^{\prime}, t, \hbar\right) d x^{\prime}-\frac{1}{2} \log \frac{\hbar P_{\text {odd }}(x, t, \hbar)}{2(x-q(t, \hbar))}\right) \\
& =\left(\frac{2(x-q(t, \hbar))}{\hbar P_{\text {odd }}(x, t, \hbar)}\right)^{1 / 2} \exp \left( \pm \int_{v}^{x} P_{\text {odd }}\left(x^{\prime}, t, \hbar\right) d x^{\prime}\right)
\end{aligned}
$$

satisfies the isomonodoromy system (2.10). Here $v$ is the simple turning point $-2 q_{0}$. The integral from $v$ is defined by

$$
\int_{v}^{x} P_{\text {odd }}\left(x^{\prime}, t, \hbar\right) d x^{\prime}=\frac{1}{2} \int_{\gamma_{x}} P_{\text {odd }}\left(x^{\prime}, t, \hbar\right) d x^{\prime},
$$

where the path $\gamma_{x}$ is depicted in Fig. 1 (cf. [26, Section 2]). 
Proof. Although the scalar version of isomonodromy system (2.10) is different from that used in [25], they are related by a gauge transformation $\psi \mapsto(x-q)^{1 / 2} \psi$. Therefore, the equalities (2.18) and (2.19) in (i) together with the holomorphicity of each coefficient of $P_{\text {odd }}(x, t, \hbar)$ at $x=q_{0}$ follows from [25, Proposition 1.2 and Theorem 1.1]. Then, it turns out that the coefficients of $P_{\text {odd }}(x, t, \hbar) /(x-q(t, \hbar))$ are also holomorphic due to (2.19). Then, (2.16) implies that each coefficient of $P_{\text {even }}(x, t, \hbar)$ is also holomorphic at $x=q_{0}$. Thus we have proved (ii).

The claim (iii) follows from a straightforward computations

$$
\begin{aligned}
\frac{1}{\psi_{ \pm}} \frac{\partial \psi_{ \pm}}{\partial x} & = \pm P_{\text {odd }}-\frac{1}{2} \frac{\partial}{\partial x} \log \left(\frac{\hbar P_{\text {odd }}}{2(x-q)}\right)= \pm P_{\text {odd }}+P_{\text {even }}=P^{( \pm)} \\
\hbar \frac{1}{\psi_{ \pm}} \frac{\partial \psi_{ \pm}}{\partial t} & =\frac{1}{2}\left(\frac{-\hbar(d q / d t)}{x-q}-\frac{\hbar}{P_{\text {odd }}} \frac{\partial P_{\text {odd }}}{\partial t}\right) \pm \int_{v}^{x} \hbar \frac{\partial P_{\text {odd }}}{\partial t} d x \\
& =-\frac{p}{2(x-q)}-\frac{\hbar}{P_{\text {odd }}}\left(\frac{1}{2(x-q)} \frac{\partial P_{\text {odd }}}{\partial x}-\frac{P_{\text {odd }}}{2(x-q)^{2}}\right) \pm \hbar \frac{P_{\text {odd }}}{2(x-q)} \\
& =-\frac{p}{2(x-q)}+\frac{\hbar P^{( \pm)}}{2(x-q)}=\frac{1}{2(x-q)}\left(\frac{\hbar}{\psi_{ \pm}} \frac{\partial \psi_{ \pm}}{\partial x}-p\right) .
\end{aligned}
$$

As we will see below, an isomonodromic WKB solution such as (2.20) is constructed from just a family of algebraic curves (2.9) by the topological recursion ([16]). In particular, the first equation in (2.10) gives a quantization of the spectral curve $(2.9)$ in the sense of $[11,12]$.

Remark 2.10. In the above computation the normalization (2.20) is essential. Since $P_{\text {odd }}$ is anti-invariant under the covering involution $\sigma$ as (2.17) and the integral in (2.20) is defined as a contour integral (2.21), we don't need to take care of the branch point $v$ in the computation

$$
\int_{v}^{x} \frac{\partial P_{\text {odd }}}{\partial t} d x=\frac{1}{2} \int_{\sigma(x)}^{x} \frac{\partial}{\partial x}\left(\frac{P_{\text {odd }}}{2(x-q)}\right) d x=\frac{P_{\text {odd }}}{2(x-q)} .
$$

Remark 2.11. We can also construct a WKB-type formal solution of matrix isomonodromy system (2.4). Define

$$
\begin{aligned}
\tilde{\psi}_{ \pm}(x, t, \hbar) & =\frac{\hbar \frac{d \psi_{ \pm}}{d x}(x, t, \hbar)-A_{11}(x, t, \hbar) \psi_{ \pm}(x, t, \hbar)}{A_{12}(x, t, \hbar)} \\
& =\frac{\hbar P^{( \pm)}(x, t, \hbar)-A_{11}(x, t, \hbar)}{A_{12}(x, t, \hbar)} \psi_{ \pm}(x, t, \hbar) .
\end{aligned}
$$

Then, the matrix valued formal series

$$
\Psi(x, t, \hbar)=\left(\begin{array}{cc}
\psi_{+}(x, t, \hbar) & \psi_{-}(x, t, \hbar) \\
\tilde{\psi}_{+}(x, t, \hbar) & \tilde{\psi}_{-}(x, t, \hbar)
\end{array}\right)
$$

gives a fundamental formal solution of the isomonodoromy system (2.4).

\section{Topological recursion and quantum curve theorem}

In this section we review the Eynard-Orantin's topological recursion [16] for our spectral curve (2.9), and formulate our main theorem. 


\subsection{Topological recursion}

The topological recursion is an algorithm associating some differential forms $W_{g, n}$ and numbers $F_{g}$ given the following source data:

- A plane curve $(\mathcal{C}, x, y): \mathcal{C}$ is a compact Riemann surface, $x, y: \mathcal{C} \rightarrow \mathbb{P}^{1}$ are meromorphic functions.

- The Bergman kernel $B$ : It is a symmetric differential form on $\mathcal{C} \times \mathcal{C}$ with poles of order 2 along the diagonal, and satisfying some normalization conditions.

In our case, $\mathcal{C}=\mathbb{P}^{1}$ and $x, y$ are rational functions which parametrize the spectral curve $(2.9)$

$$
x(z)=z^{2}-2 q_{0}, \quad y(z)=2 z\left(z^{2}-3 q_{0}\right) .
$$

Here $z$ is a coordinate on $\mathbb{P}^{1}$. The Bergman kernel is given by

$$
B\left(z_{1}, z_{2}\right)=\frac{d z_{1} d z_{2}}{\left(z_{1}-z_{2}\right)^{2}}
$$

since the spectral curve is of genus 0 . Zeros of $d x$ are called ramification points of the spectral curve (3.1). Our spectral curve has only one ramification point at $z=0$.

The topological recursion for our spectral curve (3.1) is formulated as follows (see [16] for general case):

Definition 3.1 ([16, Definition 4.2] (see also [11, Section 3])). The Eynard-Orantin differential $W_{g, n}\left(z_{1}, \ldots, z_{n}\right)$ of type $(g, n)$ is a meromorphic $n$-differential on the $n$-times product of the spectral curve (3.1) defined by the following topological recursion relation:

- for $2 g-2+n \leq 0$ :

$$
\begin{aligned}
& W_{0,1}\left(z_{1}\right):=y\left(z_{1}\right) d x\left(z_{1}\right)=4 z_{1}^{2}\left(z_{1}^{2}-3 q_{0}\right) d z_{1}, \\
& W_{0,2}\left(z_{1}, z_{2}\right):=B\left(z_{1}, z_{2}\right)=\frac{d z_{1} d z_{2}}{\left(z_{1}-z_{2}\right)^{2}},
\end{aligned}
$$

- for $2 g-2+n=1$ :

$$
\begin{aligned}
& W_{0,3}\left(z_{1}, z_{2}, z_{3}\right):=\frac{1}{2 \pi i} \oint_{\gamma_{0}} K\left(z, z_{1}\right)\left[W_{0,2}\left(z, z_{2}\right) W_{0,2}\left(\bar{z}, z_{3}\right)+W_{0,2}\left(z, z_{3}\right) W_{0,2}\left(\bar{z}, z_{2}\right)\right], \\
& W_{1,1}\left(z_{1}\right):=\frac{1}{2 \pi i} \oint_{\gamma_{0}} K\left(z, z_{1}\right) W_{0,2}(z, \bar{z})
\end{aligned}
$$

- for $2 g-2+n \geq 2$ :

$$
\begin{aligned}
& W_{g, n}\left(z_{1}, \ldots, z_{n}\right):=\frac{1}{2 \pi i} \oint_{\gamma_{0}} K\left(z, z_{1}\right) \\
& \quad \times\left[\sum_{j=2}^{n}\left(W_{0,2}\left(z, z_{j}\right) W_{g, n-1}\left(\bar{z}, z_{[\hat{i}, \hat{j}]}\right)+W_{0,2}\left(\bar{z}, z_{j}\right) W_{g, n-1}\left(z, z_{[\hat{1}, \hat{j}]}\right)\right)\right. \\
& \left.\quad+W_{g-1, n+1}\left(z, \bar{z}, z_{[\hat{1}]}\right)+\sum_{\substack{g_{1}+g_{2}=g \\
I \sqcup J=[\hat{1}]}}^{\text {stable }} W_{g_{1},|I|+1}\left(z, z_{I}\right) W_{g_{2},|J|+1}\left(\bar{z}, z_{J}\right)\right] .
\end{aligned}
$$


Here $\gamma_{0}$ is a small cycle (in $z$-plane) which encircles the ramification point $z=0$ in the counterclockwise direction, $\bar{z}=-z$ is the conjugate of $z$ near the ramification point, and the recursion kernel $K\left(z, z_{1}\right)$ is given by

$$
K\left(z, z_{1}\right)=-\frac{\omega^{\bar{z}-z}\left(z_{1}\right)}{2(y(z)-y(\bar{z})) d x(z)}, \quad \omega^{\bar{z}-z}\left(z_{1}\right)=\int_{z}^{\bar{z}} W_{0,2}\left(\cdot, z_{1}\right) .
$$

Also, we use the index convention $[\hat{j}]=\{1, \ldots, n\} \backslash\{j\}$ and so on. Lastly, the sum in the third line of (3.2) is taken for indices in the stable range (i.e., only $W_{g, n}$ 's with $2 g-2+n \geq 1$ appear).

The explicit form of some of Eynard-Orantin differentials are given as follows

$$
\begin{aligned}
& W_{0,3}=\frac{1}{12 q_{0} z_{1}^{2} z_{2}^{2} z_{3}^{2}} d z_{1} d z_{2} d z_{3}, \\
& W_{0,4}=\frac{z_{1}^{2} z_{2}^{2} z_{3}^{2} z_{4}^{2}+3 q_{0}\left(z_{1}^{2} z_{2}^{2} z_{3}^{2}+z_{2}^{2} z_{3}^{2} z_{4}^{2}+z_{3}^{2} z_{4}^{2} z_{1}^{2}+z_{4}^{2} z_{1}^{2} z_{2}^{2}\right)}{144 q_{0}^{3} z_{1}^{4} z_{2}^{4} z_{3}^{4} z_{4}^{4}} d z_{1} d z_{2} d z_{3} d z_{4}, \\
& W_{1,1}=\frac{z_{1}^{2}+3 q_{0}}{288 q_{0}^{2} z_{1}^{4}} d z_{1} \\
& W_{1,2}=\frac{2 z_{1}^{4} z_{2}^{4}+6 q_{0}\left(z_{1}^{4} z_{2}^{2}+z_{1}^{2} z_{2}^{4}\right)+3 q_{0}^{2}\left(5 z_{1}^{4}+3 z_{1}^{2} z_{2}^{2}+5 z_{2}^{4}\right)}{3456 q_{0}^{4} z_{1}^{6} z_{2}^{6}} d z_{1} d z_{2}, \\
& W_{2,1}=\frac{28 z_{1}^{8}+84 q_{0} z_{1}^{6}+252 q_{0}^{2} z_{1}^{4}+609 q_{0}^{3} z_{1}^{2}+945 q_{0}^{4}}{1990656 q_{0}^{7} z_{1}^{10}} d z_{1} .
\end{aligned}
$$

Eynard-Orantin differentials have the following properties (see [16]):

- As a differential form on each variable $z_{i}, W_{g, n}$, for $2 g-2+n \geq 1$, is holomorphic except for the ramification point 0 and may have a pole at 0 .

- $W_{g, n}$ is symmetric; that is, they are invariant under any permutation of variables.

- For $2 g-2+n \geq 1, W_{g, n}$ is anti-invariant under the involution $z_{i} \mapsto \bar{z}_{i}$ for each variable:

$$
W_{g, n}\left(z_{1}, \ldots, \bar{z}_{j}, \ldots, z_{n}\right)=-W_{g, n}\left(z_{1}, \ldots, z_{j}, \ldots, z_{n}\right) \quad \text { for } \quad j=1, \ldots, n \text {. }
$$

- $W_{g, n}$ is also holomorphic in $t$ except for $t=0$ (i.e., $q_{0}=0$ ). There is a formula for the derivative of $W_{g, n}$ with respect to $t$; see Section 3.5.

\subsection{Quantum curve theorem}

In this section we describe our main result which claims that the scalar isomonodromy system (2.10) gives a quantum curve.

Definition 3.2. For $g \geq 0, n \geq 1$ satisfying $2 g-2+n \geq 1$, define open free energy of type $(g, n)$ by

$$
F_{g, n}\left(z_{1}, \ldots, z_{n}\right):=\frac{1}{2^{n}} \int_{\bar{z}_{1}}^{z_{1}} \cdots \int_{\bar{z}_{n}}^{z_{n}} W_{g, n}\left(z_{1}, \ldots, z_{n}\right)
$$

It follows from the definition that open free energies satisfy

$$
\begin{aligned}
& d_{z_{1}} \cdots d_{z_{n}} F_{g, n}\left(z_{1}, \ldots, z_{n}\right)=W_{g, n}\left(z_{1}, \ldots, z_{n}\right), \\
& F_{g, n}\left(z_{1}, \ldots, \bar{z}_{j}, \ldots, z_{n}\right)=-F_{g, n}\left(z_{1}, \ldots, z_{j}, \ldots, z_{n}\right) \quad \text { for } \quad j=1, \ldots, n .
\end{aligned}
$$


Explicit computation shows that

$$
\begin{aligned}
& F_{0,3}\left(z_{1}, z_{2}, z_{3}\right)=-\frac{1}{12 q_{0} z_{1} z_{2} z_{3}}, \\
& F_{0,4}\left(z_{1}, z_{2}, z_{3}, z_{4}\right)=\frac{z_{1}^{2} z_{2}^{2} z_{3}^{2} z_{4}^{2}+q_{0}\left(z_{1}^{2} z_{2}^{2} z_{3}^{2}+z_{2}^{2} z_{3}^{2} z_{4}^{2}+z_{3}^{2} z_{4}^{2} z_{1}^{2}+z_{4}^{2} z_{1}^{2} z_{2}^{2}\right)}{144 q_{0}^{3} z_{1}^{3} z_{2}^{3} z_{3}^{3} z_{4}^{3}} \\
& F_{1,1}\left(z_{1}\right)=-\frac{z_{1}^{2}+q_{0}}{288 q_{0}^{2} z_{1}^{3}}, \\
& F_{1,2}\left(z_{1}, z_{2}\right)=\frac{2 z_{1}^{4} z_{2}^{4}+2 q_{0}\left(z_{1}^{4} z_{2}^{2}+z_{1}^{2} z_{2}^{4}\right)+q_{0}^{2}\left(3 z_{1}^{4}+z_{1}^{2} z_{2}^{2}+3 z_{2}^{4}\right)}{3456 q_{0}^{4} z_{1}^{5} z_{2}^{5}} \\
& F_{2,1}\left(z_{1}\right)=-\frac{140 z_{1}^{8}+140 q_{0} z_{1}^{6}+252 q_{0}^{2} z_{1}^{4}+435 q_{0}^{3} z_{1}^{2}+525 q_{0}^{4}}{9953280 q_{0}^{7} z_{1}^{9}}
\end{aligned}
$$

We also introduce functions $\left\{S_{m}(x, t)\right\}_{m \geq 0}$ by

$$
S_{0}(x, t):=\int_{v}^{x} y\left(z\left(x^{\prime}\right)\right) d x^{\prime}, \quad S_{1}(x, t):=-\frac{1}{2} \log \left(\frac{y(z(x))}{2\left(x-q_{0}\right)}\right),
$$

and for $m \geq 2$

$$
S_{m}(x, t):=\left.\sum_{\substack{2 g-2+n=m-1 \\ g \geq 0, n \geq 1}} \frac{F_{g, n}(z, \ldots, z)}{n !}\right|_{z=z(x)},
$$

where $z(x)=\sqrt{x+2 q_{0}}$ is the inverse function of $x(z)$. After computations we have

$$
\begin{aligned}
& S_{0}(x, t)=\frac{4}{5}\left(x-3 q_{0}\right)\left(x+2 q_{0}\right)^{3 / 2}, \quad S_{1}(x, t)=-\frac{1}{4} \log \left(x+2 q_{0}\right), \\
& S_{2}(x, t)=-\frac{x+7 q_{0}}{288 q_{0}^{2}\left(x+2 q_{0}\right)^{3 / 2}}, \quad S_{3}(x, t)=\frac{2 x^{2}+14 q_{0} x+35 q_{0}^{2}}{6912 q_{0}^{4}\left(x+2 q_{0}\right)^{3}} \\
& S_{4}(x, t)=-\frac{140 x^{4}+1580 q_{0} x^{3}+7476 q_{0}^{2} x^{2}+18739 q_{0}^{3} x+23499 q_{0}^{4}}{9953280 q_{0}^{7}\left(x+2 q_{0}\right)^{9 / 2}} .
\end{aligned}
$$

Our main result is the following.

Theorem 3.3. The formal series $\psi(x, t, \hbar)$ given by

$$
\begin{aligned}
\psi(x, t, \hbar) & :=\exp (S(x, t, \hbar) t), \\
S(x, t, \hbar) & :=\sum_{m=0}^{\infty} \hbar^{m-1} S_{m}(x, t)
\end{aligned}
$$

satisfies both of the differential equations in scalar-version of the isomonodromy system (2.10). That is, the formal series $S(x, t, \hbar)$ given by (3.5) satisfies the following differential equations which are equivalent to (2.10):

$$
\begin{aligned}
& \hbar^{2}\left(\left(\frac{\partial S}{\partial x}\right)^{2}+\frac{\partial^{2} S}{\partial x^{2}}\right)=\frac{\hbar}{x-q}\left(\hbar \frac{\partial S}{\partial x}-p\right)+\left(4 x^{3}+2 t x+p^{2}-4 q^{3}-2 t q\right), \\
& \hbar \frac{\partial S}{\partial t}=\frac{1}{2(x-q)}\left(\hbar \frac{\partial S}{\partial x}-p\right) .
\end{aligned}
$$


Thus, the principal specialization (i.e., setting $z_{i}=z$ for all $i=1, \ldots, n$ ) of the open free energies gives an isomonodromic WKB solution. Theorem 3.3 implies

$$
\frac{\partial S}{\partial x}(x, t, \hbar)=P^{(+)}(x, t, \hbar)
$$

holds (under a suitable choice of the branch of $\sqrt{x+2 q_{0}}$ ). The computational results in Section 2.4 show that (3.8) holds up to $\hbar^{4}$. A full proof of Theorem 3.3 will be given in Section 4 together with that of Theorem 3.7 below.

Remark 3.4. In the topological recursion (3.2), we take residues only at the ramification point $z=0$. Thus $W_{g, n}$ 's defined here are different from those in [12]; in particular, our quantum curve (2.10) has infinitely many $\hbar$-corrections as in (2.11) and (2.12) (but recovers the same spectral curve in the semi-classical limit).

Remark 3.5. In Theorem 3.3, the choice of the lower end points of the integral in (3.3) is important. Different choice also give a WKB solution of the first equation in (2.10), but it may not satisfy the second equation in general.

\subsection{Closed free energies and the $\tau$-function}

The other main result of this paper is giving another proof of the known fact about the relationship between the closed free energies and the $\tau$-function of $P_{\mathrm{I}}$ (cf. $\left.[9,16]\right)$.

Definition 3.6 ([16, Definition 4.3]). Define the closed free energy $F_{g}=F_{g}(t)$ for $g \geq 2$ by

$$
F_{g}(t)=\frac{1}{2 \pi i(2-2 g)} \oint_{\gamma_{0}} \Phi(z) W_{g, 1}(z)
$$

where

$$
\Phi(z)=\int_{z_{0}}^{z} y(z) d x(z)=\frac{4}{5} z^{5}-4 q_{0} z^{3}+\text { const }
$$

and $z_{0}$ is a generic point. Free energies $F_{0}$ and $F_{1}$ for $g=0,1$ are also defined but in a different manner (see [16, Sections 4.2.2 and 4.2.3] for the definition).

Note that $F_{g}$ defined here is different from $F_{g, n}$ defined in the previous subsection. $F_{g}$ 's are also called symplectic invariants since they are invariant under symplectic transformations of the spectral curve (see [16]). Explicit computation shows that

$$
\begin{array}{ll}
F_{0}(t)=-\frac{48 q_{0}^{5}}{5}, & F_{1}(t)=-\frac{1}{24} \log \left(-3 q_{0}\right), \\
F_{2}(t)=\frac{7}{207360 q_{0}^{5}}, & F_{3}(t)=\frac{245}{429981696 q_{0}^{10}} .
\end{array}
$$

Theorem 3.7 ([9] and [16, Section 10.6]). The generating function of the free energy $F_{g}(t)$ gives a $\tau$-function of $P_{\mathrm{I}}$ :

$$
\log \tau(t, \hbar)=\sum_{g=0}^{\infty} \hbar^{2 g-2} F_{g}(t) .
$$

Namely,

$$
\frac{d F_{g}(t)}{d t}=\sigma_{2 g}(t)
$$


The proof will be given in Section 4. It is worth mentioning that the closed free energies specify one particular $\tau$-function although there is an ambiguity in Definition 2.4.

Proposition 3.8. For $g \geq 2$, we have

$$
F_{g}(t)=\int_{\infty}^{t} \sigma_{2 g}\left(t^{\prime}\right) d t^{\prime}
$$

Proof. Let us describe the behavior of the $W_{g, n}$ 's when $q_{0} \rightarrow \infty$ (i.e., $t \rightarrow \infty$ ). When $q_{0}$ tends to $\infty$, no singular point of the integrand in the right hand-side of (3.2) on the $z$-plane hits the integration cycle $\gamma_{0}$. Thus, we can show that

$$
W_{g, n}\left(z_{1}, \ldots, z_{n}\right)=O\left(q_{0}^{-(2 g-2+n)}\right)
$$

for $2 g-2+n \geq 0$. This implies that

$$
F_{g}(t)=O\left(q_{0}^{-(2 g-2)}\right)
$$

holds since $\Phi(z) \sim q_{0}$ as $q_{0} \rightarrow \infty$ (but we can verify that $F_{g}$ for $g \geq 2$ has a stronger decay in the above explicit computations). This completes the proof of (3.10).

\subsection{Asymptotics of Eynard-Orantin differnetials}

The rest of this section will be devoted to show some important properties of $W_{g, n}$ and $F_{g, n}$. Firstly, we will describe the asymptotic behavior of them near $z_{i}=\infty$.

\section{Lemma 3.9.}

(i) For $2 g-2+n \geq 0$, we have

$$
W_{g, n}\left(z_{1}, \ldots, z_{n}\right)=\left(\frac{c_{g, n}}{z_{1}^{2} \cdots z_{n}^{2}}+O\left(z_{1}^{-4} \cdots z_{n}^{-4}\right)\right) d z_{1} \cdots d z_{n}
$$

as $z_{i} \rightarrow \infty$ for all $i=1, \ldots, n$. Here $c_{g, n} \in \mathbb{C}$ is a constant.

(ii) For $2 g-2+n \geq 0$, we have

$$
F_{g, n}\left(z_{1}, \ldots, z_{n}\right)=\frac{c_{g, n}^{\prime}}{z_{1} \cdots z_{n}}+O\left(z_{1}^{-3} \cdots z_{n}^{-3}\right), \quad c_{g, n}^{\prime} \in \mathbb{C}
$$

as $z_{i} \rightarrow \infty$ for all $i=1, \ldots, n$.

Proof. The first property (3.11) follows from the analyticity of $W_{g, n}$ at $z_{i}=\infty$. The second property (3.12) follows from (3.11) immediately because $F_{g, n}\left(z_{1}, \ldots, z_{n}\right)$ doesn't have a constant term due to the definition (3.3).

As a corollary, the principal specialization of open free energies satisfies

$$
F_{g, n}(z, \ldots, z)=O\left(z^{-n}\right)
$$

when $z \rightarrow \infty$. 


\subsection{Variation of spectral curve}

There is a formula (for "variation of spectral curves") that allows us to compute derivatives of $W_{g, n}$ etc. with respect to the parameter $t$.

Theorem 3.10 (cf. [16, Theorem 5.1]).

(i) For $2 g-2+n \geq 0$, we have

$$
\begin{aligned}
& \frac{\partial}{\partial t} W_{g, n}\left(z\left(x_{1}\right), \ldots, z\left(x_{n}\right)\right) \\
& \quad=-2 \underset{x_{n+1}=\infty}{\operatorname{Res}} z\left(x_{n+1}\right) W_{g, n+1}\left(z\left(x_{1}\right), \ldots, z\left(x_{n}\right), z\left(x_{n+1}\right)\right) .
\end{aligned}
$$

(ii) For $g \geq 1$, we have

$$
\frac{d F_{g}}{d t}(t)=-2 \underset{x=\infty}{\operatorname{Res}} z(x) W_{g, 1}(z(x))=-\operatorname{Res}_{z=\infty} z W_{g, 1}(z) .
$$

(iii) For $2 g-2+n \geq 1$, we have

$$
\begin{aligned}
& \frac{\partial}{\partial t} F_{g, n}\left(z\left(x_{1}\right), \ldots, z\left(x_{n}\right)\right) \\
& \quad=-2 \underset{x_{n+1}=\infty}{\operatorname{Res}} z\left(x_{n+1}\right) d_{x_{n+1}} F_{g, n+1}\left(z\left(x_{1}\right), \ldots, z\left(x_{n}\right), z\left(x_{n+1}\right)\right),
\end{aligned}
$$

or equivalently,

$$
\begin{aligned}
& \frac{\partial}{\partial t} F_{g, n}\left(z\left(x_{1}\right), \ldots, z\left(x_{n}\right)\right) \\
& \quad=\left.\lim _{z_{n+1} \rightarrow \infty}\left(z_{n+1}^{2} \frac{\partial}{\partial z_{n+1}} F_{g, n+1}\left(z_{1}, \ldots, z_{n}, z_{n+1}\right)\right)\right|_{\left(z_{1}, \ldots, z_{n}\right)=\left(z\left(x_{1}\right), \ldots, z\left(x_{n}\right)\right)}
\end{aligned}
$$

Proof. Set $\Lambda(z):=z$. Then, we can check $\Lambda(z)$ satisfies the required condition

$$
\underset{z=\infty}{\operatorname{Res}}\left(\Lambda(z) W_{0,2}\left(z, z_{1}\right)\right)=-d z_{1}=-\left(\frac{\partial y}{\partial t}\left(z_{1}\right) d x\left(z_{1}\right)-\frac{\partial x}{\partial t}\left(z_{1}\right) d y\left(z_{1}\right)\right)
$$

to apply [16, Theorem 5.1]. Thus the claim (i) and (ii) are proved. Integrating both hand-sides of (3.14), we have (iii).

\subsection{Differential recursion for open free energies}

Here we give a key theorem in the proof of our main results. We have the following differential recursion which is a modification of the one obtained in [11, 12].

Theorem 3.11. The open free energies for $2 g-2+n \geq 2$ satisfy the following equations

$$
\begin{aligned}
\frac{\partial F_{g, n}}{\partial z_{1}}\left(z_{1}, \ldots, z_{n}\right)= & \sum_{j=2}^{n} \frac{-2 z_{j}}{z_{1}^{2}-z_{j}^{2}}\left(\frac{1}{2 y\left(z_{1}\right) \frac{d x}{d z}\left(z_{1}\right)} \frac{\partial F_{g, n-1}}{\partial z_{1}}\left(z_{[\hat{j}]}\right)-\frac{1}{2 y\left(z_{j}\right) \frac{d x}{d z}\left(z_{j}\right)} \frac{\partial F_{g, n-1}}{\partial z_{j}}\left(z_{[\hat{1}]}\right)\right) \\
& -\frac{1}{2 y\left(z_{1}\right) \frac{d x}{d z}\left(z_{1}\right)} \frac{\partial^{2}}{\partial u_{1} \partial u_{2}}\left(F_{g-1, n+1}\left(u_{1}, u_{2}, z_{[\hat{1}]}\right)\right. \\
& \left.+\sum_{\substack{g_{1}+g_{2}=g \\
I \sqcup J=[\hat{1}]}}^{\text {stable }} F_{g_{1},|I|+1}\left(u_{1}, z_{I}\right) F_{g_{2},|J|+1}\left(u_{2}, z_{J}\right)\right)\left.\right|_{u_{1}=u_{2}=z_{1}}
\end{aligned}
$$




$$
\begin{aligned}
& +\frac{s}{\frac{d y}{d z}(s) \frac{d x}{d z}(s)\left(z_{1}^{2}-s^{2}\right)}\left[\sum_{j=2}^{n} \frac{-2 z_{j}}{z_{j}^{2}-s^{2}} \frac{\partial F_{g, n-1}}{\partial z_{1}}\left(s, z_{[\hat{1}, \hat{j}]}\right)\right. \\
& +\frac{\partial^{2}}{\partial u_{1} \partial u_{2}}\left(F_{g-1, n+1}\left(u_{1}, u_{2}, z_{[\hat{1}]}\right)\right. \\
& \left.\left.+\sum_{\substack{g_{1}+g_{2}=g \\
I \sqcup J=[\hat{1}]}}^{\text {stable }} F_{g_{1},|I|+1}\left(u_{1}, z_{I}\right) F_{g_{2},|J|+1}\left(u_{2}, z_{J}\right)\right)\left.\right|_{u_{1}=u_{2}=s}\right] .
\end{aligned}
$$

Here $s=\left(3 q_{0}\right)^{1 / 2}$ is a zero of $y(z)$.

Proof. This can be proved by a similar technique used in [11, Theorem 4.7], as follows. Integrating the topological recursion relation (3.2) with respect to $z_{2}, \ldots, z_{n}$, we have

$$
\begin{aligned}
\frac{\partial}{\partial z_{1}} F_{g, n}\left(z_{1}, \ldots, z_{n}\right) & =\frac{1}{2^{n-1}} \int_{\bar{z}_{2}}^{z_{2}} \cdots \int_{\bar{z}_{n}}^{z_{n}} W_{g, n}\left(z_{1}, \ldots, z_{n}\right) \\
& =\frac{1}{2 \pi i} \frac{1}{2^{n-1}} \oint_{\gamma_{0}} K\left(z, z_{1}\right) R_{g, n}\left(z, z_{2}, \ldots, z_{n}\right),
\end{aligned}
$$

where

$$
\begin{aligned}
R_{g, n}\left(z, z_{2}, \ldots, z_{n}\right)= & \sum_{j=2}^{n}\left[\left(\int_{\bar{z}_{j}}^{z_{j}} W_{0,2}\left(z, z_{j}\right)\right)\left(\int_{\bar{z}_{[\hat{i}, \hat{j}]}}^{z_{[\hat{1}, \hat{j}]}} W_{g, n-1}\left(\bar{z}, z_{[\hat{1}, \hat{j}]}\right)\right)\right. \\
& \left.-\left(\int_{\bar{z}_{j}}^{z_{j}} W_{0,2}\left(\bar{z}, z_{j}\right)\right)\left(\int_{\bar{z}_{[\hat{1}, \hat{j}]}}^{z_{[\hat{i}, \hat{j}]}} W_{g, n-1}\left(z, z_{[\hat{1}, \hat{j}]}\right)\right)\right] \\
& +\int_{\bar{z}_{[\hat{1}]}}^{z_{[\hat{1}]}} W_{g-1, n+1}\left(z, \bar{z}, z_{[\hat{1}]}\right) \\
& +\sum_{\substack{g_{1}+g_{2}=g \\
I \sqcup J=[\hat{1}]}}^{\operatorname{stable}}\left(\int_{\bar{z}_{I}}^{z_{I}} W_{g_{1},|I|+1}\left(z, z_{I}\right)\right)\left(\int_{\bar{z}_{J}}^{z_{J}} W_{g_{2},|J|+1}\left(\bar{z}, z_{J}\right)\right) .
\end{aligned}
$$

Here, for a set $L=\left\{\ell_{1}, \ldots, \ell_{k}\right\} \subset\{1, \ldots, n\}$ of indices, we have used the notation

$$
\int_{\bar{z}_{L}}^{z_{L}} W_{g, n}\left(z_{1}, \ldots, z_{n}\right):=\int_{\bar{z}_{\ell_{1}}}^{z_{\ell_{1}}} \cdots \int_{\bar{z}_{\ell_{k}}}^{z_{\ell_{k}}} W_{g, n}\left(z_{1}, \ldots, z_{n}\right) .
$$

On the $z$-plane, the integrand $K\left(z, z_{1}\right) R_{g, n}\left(z, z_{1}, \ldots, z_{n}\right)$ in the right hand-side of (3.18) has poles at

- at $z=z_{1}, \bar{z}_{1}$ which are poles of $K\left(z, z_{1}\right)$,

- at $z=z_{2}, \ldots, z_{n}, \bar{z}_{2}, \ldots, \bar{z}_{n}$ which are poles of $W_{0,2}\left(z, z_{j}\right)$ and $W_{0,2}\left(\bar{z}, z_{j}\right)$,

- at $z=s, \bar{s}$ which are poles of $K\left(z, z_{1}\right)$,

and all of them are simple poles. Then, the equalities

$$
\begin{aligned}
& \int_{\bar{z}_{j}}^{z_{j}} W_{0,2}\left(z, z_{j}\right)=\left(\frac{1}{z-z_{j}}-\frac{1}{z-\bar{z}_{j}}\right) d z, \\
& \frac{1}{2^{n-2}} \int_{\bar{z}_{[\hat{[}, \hat{j}]}}^{z_{[\hat{1}, \hat{j}]}} W_{g, n}\left(z, z_{[\hat{1}, \hat{j}]}\right)=\frac{\partial F_{g, n-1}}{\partial z_{1}}\left(z, z_{[\hat{i}, \hat{j}]}\right)
\end{aligned}
$$

and the residue theorem show (3.17). 
Remark 3.12. Note that the first two blocks in the right hand-side of (3.17) coincide with that obtained in $[11,12]$. Unlike the case of $[11,12]$, we need more terms arising from $z=s$ corresponding to the singular point $(x, y)=\left(q_{0}, 0\right)$ of the spectral curve $(2.9)$ since it becomes a (simple) pole of the recursion kernel $K\left(z, z_{1}\right)$. It also worth mentioning that the right hand-side of (3.17) doesn't have singularity at $z_{j}=s$ for $j=1, \ldots, n$.

Using this differential recursion, we can give an alternative expression of (3.16) as follows.

Theorem 3.13. For $2 g-2+n \geq 1$, the following holds:

$$
\frac{\partial}{\partial t} F_{g, n}\left(z\left(x_{1}\right), \ldots, z\left(x_{n}\right)\right)=E_{g, n}\left(z\left(x_{1}\right), \ldots, z\left(x_{n}\right)\right),
$$

where

$$
\begin{aligned}
E_{g, n}\left(z_{1}, \ldots, z_{n}\right) & \\
:= & \sum_{j=1}^{n} \frac{2 z_{j}}{2 y\left(z_{j}\right) \frac{d x}{d z}\left(z_{j}\right)} \frac{\partial F_{g, n}}{\partial z_{j}}\left(z_{1}, \ldots, z_{n}\right)+\left.\frac{s}{\frac{d y}{d z}(s) \frac{d x}{d z}(s)} \sum_{j=1}^{n} \frac{-2 z_{j}}{z_{j}^{2}-s^{2}} \frac{\partial F_{g, n}}{\partial u_{1}}\left(u_{1}, z_{[\hat{j}]}\right)\right|_{u_{1}=s} \\
& +\frac{s}{\frac{d y}{d z}(s) \frac{d x}{d z}(s)} \frac{\partial^{2}}{\partial u_{1} \partial u_{2}}\left(F_{g-1, n+2}\left(u_{1}, u_{2}, z_{1}, \ldots, z_{n}\right)\right. \\
& \left.+\sum_{\substack{g_{1}+g_{2}=g \\
I \sqcup J=\{1, \ldots, n\}}}^{\text {stable }} F_{g_{1},|I|+1}\left(u_{1}, z_{I}\right) F_{g_{2},|J|+1}\left(u_{2}, z_{J}\right)\right)\left.\right|_{u_{1}=u_{2}=s}
\end{aligned}
$$

Proof. The equality (3.16) shows that the left hand-side of (3.19) coincides with

$$
\lim _{z_{n+1} \rightarrow \infty} z_{n+1}^{2} \frac{\partial}{\partial z_{n+1}} F_{g, n+1}\left(z_{1}, \ldots, z_{n}, z_{n+1}\right)
$$

after the substitution $z_{i} \mapsto z\left(x_{i}\right)$ for $i=1, \ldots, n$. Then, the equality follows from the asymptotic behavior (3.12) of $F_{g, n}$ 's and the above differential recursion $(3.17)$ for $2 g-2+(n+1) \geq 2$.

\section{Proof of main theorems}

\subsection{Strategy for the proof}

What we will show here is that the formal series $S(x, t, \hbar)$ defined in $(3.5)$ satisfies the system of equations (3.6) and (3.7). In addition, we will also prove the equality (3.9). These equalities will be proved by an induction as follows.

Theorem 4.1. Let $[\bullet]_{\hbar^{m}}$ be the coefficient of $\hbar^{m}$ in a formal series $\bullet$ of $\hbar$. For an even integer $k \geq 2$, assume that

$$
\begin{aligned}
& \frac{\partial S_{m}}{\partial x}(x, t)=P_{m}(x, t) \quad \text { for } \quad m=0, \ldots, k-1, \\
& \frac{\partial S_{m}}{\partial t}(x, t)=\left[\frac{1}{2(x-q)}\left(\hbar \frac{\partial S}{\partial x}-p\right)\right]_{\hbar^{m}} \quad \text { for } \quad m=0, \ldots, k-1, \\
& \frac{d F_{g}}{d t}(t)=\sigma_{2 g}(t) \quad \text { for } \quad g=k / 2
\end{aligned}
$$

holds. Here $P_{m}(x, t)=P_{m}^{(+)}(x, t)$ is the coefficient of $\hbar^{m-1}$ in the formal solution $P^{(+)}(x, t, \hbar)$ of the Riccati equation (2.13) constructed in Section 2.4, and $\sigma_{2 g}$ is given in (2.7). Then, we have 
(A) The following equality holds for $m=k$ and $k+1$ :

$$
\left[\hbar^{2}\left(\left(\frac{\partial S}{\partial x}\right)^{2}+\frac{\partial^{2} S}{\partial x^{2}}\right)\right]_{\hbar^{m}}=\left[2 \hbar^{2} \frac{\partial S}{\partial t}+\left(4 x^{3}+2 t x+p^{2}-4 q^{3}-2 t q\right)\right]_{\hbar^{m}} .
$$

(B) The following equalities hold:

$$
\begin{aligned}
& \frac{\partial S_{k}}{\partial x}(x, t)=P_{k}(x, t), \quad \frac{\partial S_{k}}{\partial t}(x, t)=\left[\frac{1}{2(x-q)}\left(\hbar \frac{\partial S}{\partial x}-p\right)\right]_{\hbar^{k}}, \\
& \frac{\partial S_{k+1}}{\partial x}(x, t)=P_{k+1}(x, t), \quad \frac{\partial S_{k+1}}{\partial t}(x, t)=\left[\frac{1}{2(x-q)}\left(\hbar \frac{\partial S}{\partial x}-p\right)\right]_{\hbar^{k+1}}, \\
& \frac{d F_{g}}{d t}(t)=\sigma_{2 g}(t) \quad \text { for } \quad g=(k+2) / 2 .
\end{aligned}
$$

It is obvious that our main theorems (Theorems 3.3 and 3.7) follow from the statements in $(A)$ and $(B)$. The rest of this section is devoted to give a proof of $(A)$ and $(B)$.

\subsection{Proof of $(A)$}

We emphasize that the results shown in Section 4.2.1 below are proved without using the assumption (4.1). We also note that we only use the second equality in assumption (4.1) in Section 4.2 .2 to prove $(A)$.

\subsubsection{Computation of principal specializations}

Define

$$
\begin{aligned}
G_{g, n}\left(z_{1}, \ldots, z_{n}\right):= & \frac{\partial F_{g, n}}{\partial z_{1}}\left(z_{1}, \ldots, z_{n}\right)-\sum_{j=2}^{n} \frac{-2 z_{j}}{z_{1}^{2}-z_{j}^{2}}\left(\frac{1}{2 y\left(z_{1}\right) \frac{d x}{d z}\left(z_{1}\right)} \frac{\partial F_{g, n-1}}{\partial z_{1}}\left(z_{[\hat{j}]}\right)\right. \\
& \left.-\frac{1}{2 y\left(z_{j}\right) \frac{d x}{d z}\left(z_{j}\right)} \frac{\partial F_{g, n-1}}{\partial z_{j}}\left(z_{[\hat{1}]}\right)\right) \\
& +\frac{1}{2 y\left(z_{1}\right) \frac{d x}{d z}\left(z_{1}\right)} \frac{\partial^{2}}{\partial u_{1} \partial u_{2}}\left(F_{g-1, n+1}\left(u_{1}, u_{2}, z_{[\hat{1}]}\right)\right. \\
& \left.+\sum_{\substack{g_{1}+g_{2}=g \\
I \sqcup J=[\hat{1}]}}^{\text {stable }} F_{g_{1},|I|+1}\left(u_{1}, z_{I}\right) F_{g_{2},|J|+1}\left(u_{2}, z_{J}\right)\right)\left.\right|_{u_{1}=u_{2}=z_{1}} .
\end{aligned}
$$

The technique developed in $[11,12]$ enables us to show the following.

Lemma 4.2 (cf. [11, Theorem 6.5]). For $m \geq 2$, we have

$$
\left.\left(\frac{2 y(z)}{\frac{d x}{d z}(z)} \sum_{\substack{2 g-2+n=m \\ g \geq 0, n \geq 1}} \frac{G_{g, n}(z, \ldots, z)}{(n-1) !}\right)\right|_{z=z(x)}=\sum_{\substack{a+b=m+1 \\ a, b \geq 0}} \frac{\partial S_{a}}{\partial x} \frac{\partial S_{b}}{\partial x}+\frac{\partial^{2} S_{m}}{\partial x^{2}}-\frac{1}{x-q_{0}} \frac{\partial S_{m}}{\partial x} .
$$

Proof. As is shown in [11, Theorem 6.5], applying $\sum_{2 g-2+n=m} \frac{1}{(n-1) !}$ and the principal specialization to (4.6), we have

$$
\sum_{\substack{2 g-2+n=m \\ g \geq 0, n \geq 1}} \frac{G_{g, n}(z, \ldots, z)}{(n-1) !}=\frac{1}{2 y(z) \frac{d x}{d z}(z)}\left(\sum_{\substack{a+b=m+1 \\ a, b \geq 2}} \frac{\partial S_{a}(x(z))}{\partial z} \frac{\partial S_{b}(x(z))}{\partial z}+\frac{\partial^{2} S_{m}(x(z))}{\partial z^{2}}\right)
$$




$$
+\frac{\partial S_{m+1}(x(z))}{\partial z}+\left\{\frac{\partial}{\partial z}\left(\frac{1}{2 y(z) \frac{d x}{d z}(z)}\right)\right\} \frac{\partial S_{m}(x(z))}{\partial z} .
$$

After the coordinate change $z=z(x)$, the right hand-side becomes

$$
\frac{\frac{d x}{d z}(z(x))}{2 y(z(x))}\left(\sum_{\substack{a+b=m+1 \\ a, b \geq 2}} \frac{\partial S_{a}}{\partial x} \frac{\partial S_{b}}{\partial x}+\frac{\partial^{2} S_{m}}{\partial x^{2}}+2 y(z(x)) \frac{\partial S_{m+1}}{\partial x}-\frac{1}{y(z(x))} \frac{\partial y(z(x))}{\partial x} \frac{\partial S_{m}}{\partial x}\right) .
$$

Then, the desired equality (4.7) follows from the above equality and

$$
\frac{\partial S_{0}}{\partial x}=y(z(x)), \quad \frac{\partial S_{1}}{\partial x}=-\frac{1}{2 y(z(x))} \frac{\partial y(z(x))}{\partial x}+\frac{1}{2\left(x-q_{0}\right)} .
$$

Note that the right hand-side of (4.7) coincides with

$$
\left[\hbar^{2}\left(\left(\frac{\partial S}{\partial x}\right)^{2}+\frac{\partial^{2} S}{\partial x^{2}}\right)\right]_{\hbar^{m+1}}-\frac{1}{x-q_{0}} \frac{\partial S_{m}}{\partial x} .
$$

Thus, Lemma 4.2 relates the principal specialization of $G_{g, n}$ to the left hand-side of (4.2). Next we also relate them to the right hand-side of (4.2).

Lemma 4.3. Let $E_{g, n}\left(z_{1}, \ldots, z_{n}\right)$ be the functions defined by (3.20). Then, the following equality holds for $m \geq 2$

$$
\left.\sum_{\substack{2 g-2+n=m \\ g \geq 0, n \geq 2}}\left(\frac{2 y(z)}{\frac{d x}{d z}(z)} \frac{G_{g, n}(z, \ldots, z)}{(n-1) !}-\frac{2 E_{g, n-1}(z, \ldots, z)}{(n-1) !}\right)\right|_{z=z(x)}=-\frac{1}{x-q_{0}} \frac{\partial S_{m}}{\partial x} .
$$

Proof. Theorem 3.11 shows that (4.6) can also be written as

$$
\begin{aligned}
G_{g, n}\left(z_{1}, \ldots, z_{n}\right)= & \frac{s}{\frac{d y}{d z}(s) \frac{d x}{d z}(s)\left(z_{1}^{2}-s^{2}\right)} \sum_{j=2}^{n} \frac{-2 z_{j}}{z_{j}^{2}-s^{2}} \frac{\partial F_{g, n-1}}{\partial z_{1}}\left(s, z_{[\hat{1}, \hat{j}]}\right) \\
& +\frac{s}{\frac{d y}{d z}(s) \frac{d x}{d z}(s)\left(z_{1}^{2}-s^{2}\right)} \frac{\partial^{2}}{\partial u_{1} \partial u_{2}}\left(F_{g-1, n+1}\left(u_{1}, u_{2}, z_{[\hat{1}]}\right)\right. \\
& \left.+\sum_{\substack{g_{1}+g_{2}=g \\
I \sqcup J=[\hat{1}]}}^{\text {stable }} F_{g_{1},|I|+1}\left(u_{1}, z_{I}\right) F_{g_{2},|J|+1}\left(u_{2}, z_{J}\right)\right)\left.\right|_{u_{1}=u_{2}=s} .
\end{aligned}
$$

Taking the principal specialization of (4.9) and (3.20) with $n \mapsto n-1$, we have

$$
\begin{aligned}
& \frac{2 y(z)}{\frac{d x}{d z}(z)} \frac{G_{g, n}(z, \ldots, z)}{(n-1) !}-\frac{2 E_{g, n-1}(z, \ldots, z)}{(n-1) !} \\
&=-\frac{4 z}{2 y(z) \frac{d x}{d z}(z)}\left(\frac{1}{(n-1) !} \frac{\partial}{\partial z} F_{g, n-1}(z, \ldots, z)\right)
\end{aligned}
$$

for any $g \geq 0$ and $n \geq 2$ satisfying $2 g-2+n \geq 2$. Then, summing up (4.10) for $g \geq 0, n \geq 2$ satisfying $2 g-2+n=m$, we obtain (4.8) after the coordinate change $z=z(x)$.

On the other hand, Theorem 3.13 implies that

$$
\left.\sum_{\substack{2 g-2+n=m \\ g \geq 0, n \geq 2}} \frac{2 E_{g, n-1}(z, \ldots, z)}{(n-1) !}\right|_{z=z(x)}=2 \frac{\partial}{\partial t} S_{m}=\left[2 \hbar^{2} \frac{\partial S}{\partial t}\right]_{\hbar^{m+1}}
$$

holds for $m \geq 2$. Therefore we have the following. 
Lemma 4.4. The equality

$$
\begin{aligned}
& {\left[\hbar^{2}\left(\left(\frac{\partial S}{\partial x}\right)^{2}+\frac{\partial^{2} S}{\partial x^{2}}\right)\right]_{\hbar^{m+1}}} \\
& \quad=\left[2 \hbar^{2} \frac{\partial S}{\partial t}\right]_{\hbar^{m+1}}+\sum_{\substack{2 g-2+n=m \\
g \geq 0, n \geq 1}} \frac{2 y(z)}{d z} \frac{G_{g, n}(z, \ldots, z)}{(n-1) !}-\sum_{\substack{2 g-2+n=m \\
g \geq 0, n \geq 2}} \frac{2 y(z)}{d z} \frac{G_{g, n}(z, \ldots, z)}{(n-1) !}
\end{aligned}
$$

holds for $m \geq 2$.

\subsubsection{Completion of the proof of $(A)$}

Lemma 4.4 implies

$$
\begin{aligned}
& {\left[\hbar^{2}\left(\left(\frac{\partial S}{\partial x}\right)^{2}+\frac{\partial^{2} S}{\partial x^{2}}\right)\right]_{\hbar^{m+1}}=\left[2 \hbar^{2} \frac{\partial S}{\partial t}\right]_{\hbar^{m+1}} \quad \text { if } m \text { is even, }} \\
& {\left[\hbar^{2}\left(\left(\frac{\partial S}{\partial x}\right)^{2}+\frac{\partial^{2} S}{\partial x^{2}}\right)\right]_{\hbar^{m+1}}=\left[2 \hbar^{2} \frac{\partial S}{\partial t}\right]_{\hbar^{m+1}}+\frac{2 y}{\frac{d x}{d z}} G_{(m+1) / 2,1} \quad \text { if } m \text { is odd. }}
\end{aligned}
$$

On the other hand, it follows from (2.7) that

$$
\left[4 x^{3}+2 t x+p^{2}-4 q^{3}-2 t q\right]_{\hbar^{m+1}}= \begin{cases}0 & \text { if } m \text { is even } \\ 2 \sigma_{m+1} & \text { if } m \text { is odd }\end{cases}
$$

Therefore, under the assumption (4.1), the desired equality (4.2) follows from (4.11) and Lemma 4.5 below.

Lemma 4.5. For $g \geq 2$, we have

$$
\frac{2 y(z)}{\frac{d x}{d z}(z)} G_{g, 1}(z)=2 \frac{d F_{g}}{d t}(t) .
$$

Proof. Firstly, we note that

$$
\frac{2 y(z)}{\frac{d x}{d z}(z)} G_{g, 1}(z)=\frac{1}{4 s^{2}}\left(\frac{\partial^{2} F_{g-1,2}}{\partial z_{1} \partial z_{2}}(s, s)+\sum_{\substack{g_{1}+g_{2}=g \\ g_{1}, g_{2} \geq 1}} \frac{\partial F_{g_{1}, 1}}{\partial z_{1}}(s) \frac{\partial F_{g_{2}, 1}}{\partial z_{2}}(s)\right)
$$

holds. Using the differnetial recursion (3.17) for $n=1$, we have

$$
\begin{aligned}
\frac{\partial F_{g, 1}}{\partial z_{1}}(z)= & -\frac{1}{2 y(z) \frac{d x}{d z}(z)}\left(\frac{\partial^{2} F_{g-1,2}}{\partial z_{1} \partial z_{2}}(z, z)+\sum_{\substack{g_{1}+g_{2}=g \\
g_{1}, g_{2} \geq 1}} \frac{\partial F_{g_{1}, 1}}{\partial z_{1}}(z) \frac{\partial F_{g_{2}, 1}}{\partial z_{1}}(z)\right) \\
& +\frac{s}{\frac{d y}{d z}(s) \frac{d x}{d z}(s)\left(z^{2}-s^{2}\right)}\left(\frac{\partial^{2} F_{g-1,2}}{\partial z_{1} \partial z_{2}}(s, s)+\sum_{\substack{g_{1}+g_{2}=g \\
g_{1}, g_{2} \geq 1}} \frac{\partial F_{g_{1}, 1}}{\partial z_{1}}(s) \frac{\partial F_{g_{2}, 1}}{\partial z_{1}}(s)\right) .
\end{aligned}
$$

Then, Lemma 3.9 implies that

$$
z \frac{\partial F_{g, 1}}{\partial z_{1}}(z) d z=z W_{g, 1}(z)=\frac{1}{8 s^{2}}\left(\frac{\partial^{2} F_{g-1,2}}{\partial z_{1} \partial z_{2}}(s, s)+\sum_{\substack{g_{1}+g_{2}=g \\ g_{1}, g_{2} \geq 1}} \frac{\partial F_{g_{1}, 1}}{\partial z_{1}}(s) \frac{\partial F_{g_{2}, 1}}{\partial z_{2}}(s)\right) \frac{d z}{z}+O(1)
$$

holds when $z \rightarrow \infty$. Then the equality (4.12) follows from (3.15) and (4.13). 


\subsection{Proof of $(B)$}

One of the desired equality (4.3) is proved as follows.

Lemma 4.6. Under the assumption (4.1), we have

$$
\begin{aligned}
& \frac{\partial S_{k}}{\partial x}(x, t)=P_{k}(x, t), \\
& S_{k}(x, t)=\int_{\infty}^{x} P_{k}\left(x^{\prime}, t\right) d x^{\prime}, \\
& \frac{\partial S_{k}}{\partial t}(x, t)=\left[\frac{1}{2(x-q)}\left(\hbar \frac{\partial S}{\partial x}-p\right)\right]_{\hbar^{k}} .
\end{aligned}
$$

Proof. The equality (4.2) for $m=k$ and the second equality in the assumption (4.1) imply

$$
\left[\hbar^{2}\left(\left(\frac{\partial S}{\partial x}\right)^{2}+\frac{\partial^{2} S}{\partial x^{2}}\right)\right]_{\hbar^{k}}=\left[\frac{\hbar}{x-q}\left(\hbar \frac{\partial S}{\partial x}-p\right)+\left(4 x^{3}+2 t x+p^{2}-4 q^{3}-2 t q\right)\right]_{\hbar^{k}} .
$$

Thus $\partial S_{k} / \partial x$ and $P_{k}$ satisfy the same equation (2.14) under our induction hypothesis. Then the uniqueness of the solution of (2.14) implies (4.14).

Since $S_{m}(x)$ for $m \geq 2$ decay when $x \rightarrow \infty$ (cf. (3.13)), the equality (4.15) immediately follows from (4.14). Then, the equality (2.18) shows

$$
\frac{\partial}{\partial t} S_{k}(x, t)=\int_{\infty}^{x}\left[\hbar \frac{\partial}{\partial t} P\right]_{\hbar^{k}} d x=\int_{\infty}^{x} \frac{\partial}{\partial x}\left[\frac{\hbar P-p}{2(x-q)}\right]_{\hbar^{k}} d x=\left[\frac{1}{2(x-q)}\left(\hbar \frac{\partial S}{\partial x}-p\right)\right]_{\hbar^{k}} .
$$

The last equality follows from the assumption (4.1) and the fact that $P_{m}(x, t)$ 's decay when $x \rightarrow \infty$ for $m \geq 1$ (see Remark 2.8), and

$$
\lim _{x \rightarrow \infty} \frac{P_{0}(x, t)}{\left(x-q_{0}\right)^{2}}=0 .
$$

Thus we have proved (4.16).

Since we have also already proved (4.2) for $m=k+1$, we can prove (4.4) by the same discussion as the proof of Lemma 4.6 above. Then, finally we obtain

Lemma 4.7. The equality (4.5) is true; namely, we have

$$
\frac{d F_{(k+2) / 2}}{d t}=\sigma_{2 k+2}
$$

Proof. It follows from the equality (4.11) (for the odd number $m=k+1$ ) and Lemma 4.5 that

$$
2 \frac{\partial S_{0}}{\partial x} \frac{\partial S_{k+2}}{\partial x}+\sum_{\substack{a+b=k+2 \\ a, b \geq 1}} \frac{\partial S_{a}}{\partial x} \frac{\partial S_{b}}{\partial x}+\frac{\partial^{2} S_{k+1}}{\partial x^{2}}-2 \frac{\partial S_{k+1}}{\partial t}=2 \frac{d F_{(k+2) / 2}}{d t}
$$

holds. On the other hand, we know that $P_{k+2}$ satisfies

$$
2 P_{0} P_{k+2}+\sum_{\substack{a+b=k+2 \\ a, b \geq 1}} P_{a} P_{b}+\frac{\partial P_{k+1}}{\partial x}-\left[\frac{\hbar}{x-q}(\hbar P-p)\right]_{\hbar^{k+2}}=2 \sigma_{k+2}
$$

(cf. (2.14)). Under our assumption, comparing (4.18) and (4.19), we have

$$
\frac{\partial S_{0}}{\partial x}\left(\frac{\partial S_{k+2}}{\partial x}-P_{k+2}\right)=\frac{d F_{(k+2) / 2}}{d t}-\sigma_{k+2} .
$$


Note that the right hand-side doesn't depend on $x$. Then, thanks to the fact

$$
\left.\frac{\partial S_{0}}{\partial x}\right|_{x=q_{0}}=0
$$

and the holomorphicity of $S_{m}(x)$ and $P_{m}(x)$ at the double turning point $x=q_{0}$ (see Theorem 2.9), we have the desired equality (4.17) by substituting $x=q_{0}$ into (4.20).

This completes the proof of $(B)$ and Theorem 4.1. Thus we have proved Theorems 3.3 and 3.7.

Remark 4.8. Since the spectral curve (2.9) has only one branch point, we have

$$
\int_{v}^{x} P_{m}\left(x^{\prime}, t\right) d x^{\prime}=\int_{\infty}^{x} P_{m}\left(x^{\prime}, t\right) d x^{\prime}
$$

for all even $m \geq 2$. This implies that the WKB solution (3.4) defined by the topological recursion coincides with the WKB solution (2.20) constructed in Section 2.4. However, the above equality (4.21) may not hold for other Painlevé equations since their spectral curves have more branch points in general.

\section{A Alternative definition of the $\tau$-function by Jimbo-Miwa-Ueno}

There is another definition of $\tau$-function (2.8) in terms of the formal solution (2.22) of the isomonodromy system.

Proposition A.1 ([20, Section 5]; see also [5, Section 4.2] and [7, Section 1.5]). The $\tau$-function satisfies

$$
\frac{d}{d t} \log \tau(t, \hbar)=-2 \operatorname{Res}_{x=\infty}\left(\frac{1}{\hbar} \frac{\partial T_{\infty}}{\partial t}(x, t) \mathcal{W}_{1}(x, t, \hbar) d x\right),
$$

where

$$
T_{\infty}(x, t):=\frac{4 x^{5 / 2}}{5}+t x^{1 / 2}
$$

(which is the divergent part of $\int^{x} P_{0}^{(+)}\left(x^{\prime}, t\right) d x^{\prime}$ as $\left.x \rightarrow \infty\right)$, and

$$
\mathcal{W}_{1}(x, t, \hbar)=\frac{\partial \psi_{+}}{\partial x}(x, t, \hbar) \tilde{\psi}_{-}(x, t, \hbar)-\frac{\partial \tilde{\psi}_{+}}{\partial x}(x, t, \hbar) \psi_{-}(x, t, \hbar)
$$

Proof. It follows from the definition (2.22) of $\Psi$ that

$$
\mathcal{W}_{1}(x, t, \hbar)=P^{(+)}(x, t, \hbar)+\frac{A_{12}(x, t, \hbar)}{2 \hbar P_{\text {odd }}(x, t, \hbar)} \frac{\partial}{\partial x}\left(\frac{\hbar P^{(+)}(x, t, \hbar)-A_{11}(x, t, \hbar)}{A_{12}(x, t, \hbar)}\right)
$$

Then, the asymptotics $(2.15)$ of $P^{( \pm)}(x, t, \hbar)$ implies that

$$
\mathcal{W}_{1}(x, t, \hbar)=\frac{2}{\hbar} x^{3 / 2}+\frac{t}{2 \hbar} x^{-1 / 2}+\frac{\sigma(t, \hbar)}{2 \hbar} x^{-3 / 2}+O\left(x^{-2}\right)
$$

holds when $x \rightarrow \infty$, and thus we have (A.1). 


\section{Acknowledgements}

The authors are grateful to Motohico Mulase for many valuable comments, discussion and continuous encouragements. They also thank Olivia Dumitrescu and Bertrand Eynard for helpful comments. K.I. work is supported by the JSPS for Advancing Strategic International Networks to Accelerate the Circulation of Talented Researchers "Mathematical Science of Symmetry, Topology and Moduli, Evolution of International Research Network based on Osaka City University Advanced Mathematical Institute (OCAMI)". A.S. work is supported by UC Davis under the Graduate Research Mentorship fellowship. This article is written during the K.I. stay at The University of California, Davis. K.I. would also like to thank the institute for its support and hospitality.

\section{References}

[1] Aganagic M., Cheng M.C.N., Dijkgraaf R., Krefl D., Vafa C., Quantum geometry of refined topological strings, J. High Energy Phys. 2012 (2012), no. 11, 019, 53 pages, arXiv:1105.0630.

[2] Aganagic M., Dijkgraaf R., Klemm A., Mariño M., Vafa C., Topological strings and integrable hierarchies, Comm. Math. Phys. 261 (2006), 451-516, hep-th/0312085.

[3] Aoki T., Honda N., Umeta Y., On a construction of general formal solutions for equations of the first Painlevé hierarchy I, Adv. Math. 235 (2013), 496-524.

[4] Aoki T., Kawai T., Takei Y., WKB analysis of Painlevé transcendents with a large parameter. II. Multiplescale analysis of Painlevé transcendents, in Structure of Solutions of Differential Equations (Katata/Kyoto, 1995), World Sci. Publ., River Edge, NJ, 1996, 1-49.

[5] Bergére M., Borot G., Eynard B., Rational differential dystems, loop equations, and application to the $q$ th reductions of KP, Ann. Henri Poincaré 16 (2015), 2713-2782, arXiv:1312.4237.

[6] Bergére M., Eynard B., Determinantal formulae and loop equations, arXiv:0901.3273.

[7] Borot G., Eynard B., Tracy-Widom GUE law and symplectic invariants, arXiv:1011.1418.

[8] Costin O., Asymptotics and Borel summability, Chapman \& Hall/CRC Monographs and Surveys in Pure and Applied Mathematics, Vol. 141, CRC Press, Boca Raton, FL, 2009.

[9] Di Francesco P., Ginsparg P., Zinn-Justin J., 2D gravity and random matrices, Phys. Rep. 254 (1995), 1-133, hep-th/9306153.

[10] Dijkgraaf R., Fuji H., Manabe M., The volume conjecture, perturbative knot invariants, and recursion relations for topological strings, Nuclear Phys. B 849 (2011), 166-211, arXiv:1010.4542.

[11] Dumitrescu O., Mulase M., Quantum curves for Hitchin fibrations and the Eynard-Orantin theory, Lett. Math. Phys. 104 (2014), 635-671, arXiv:1310.6022.

[12] Dumitrescu O., Mulase M., Quantization of spectral curves for meromorphic Higgs bundles through topological recursion, arXiv:1411.1023.

[13] Dunin-Barkowski P., Mulase M., Norbury P., Popolitov A., Shadrin S., Quantum spectral curve for the Gromov-Witten theory of the complex projective line, J. Reine Angew. Math., to appear, arXiv:1312.5336.

[14] Eynard B., Topological recursion and quantum curves, Talk given in the workshop "Quantum curves, Hitchin systems, and the Eynard-Orantin theory", American Institute of Mathematics, Palo Alto, September 2014.

[15] Eynard B., Counting surfaces, Progress in Mathematical Physics, Vol. 70, Birkhäuser, Basel, 2016.

[16] Eynard B., Orantin N., Invariants of algebraic curves and topological expansion, Commun. Number Theory Phys. 1 (2007), 347-452, math-ph/0702045.

[17] Fokas A.S., Its A.R., Kapaev A.A., Novokshenov V.Yu., Painlevé transcendents. The Riemann-Hilbert approach, Mathematical Surveys and Monographs, Vol. 128, Amer. Math. Soc., Providence, RI, 2006.

[18] Gukov S., Sułkowski P., A-polynomial, B-model, and quantization, J. High Energy Phys. 2012 (2012), no. 2, 070, 57 pages, arXiv:1108.0002.

[19] Iwaki K., Marchal O., Painlevé 2 equation with arbitrary monodromy parameter, topological recursion and determinantal formulas, arXiv:1411.0875.

[20] Jimbo M., Miwa T., Ueno K., Monodromy preserving deformation of linear ordinary differential equations with rational coefficients. I. General theory and $\tau$-function, Phys. D 2 (1981), 306-352. 
[21] Jimbo M., Miwa T., Monodromy preserving deformation of linear ordinary differential equations with rational coefficients. II, Phys. D 2 (1981), 407-448.

[22] Joshi N., Kitaev A.V., On Boutroux's tritronquée solutions of the first Painlevé equation, Stud. Appl. Math. 107 (2001), 253-291.

[23] Kamimoto S., Koike T., On the Borel summability of 0-parameter solutions of nonlinear ordinary differential equations, in Recent Development of Micro-Local Analysis for the Theory of Asymptotic Analysis, RIMS Kôkyûroku Bessatsu, Vol. B40, Res. Inst. Math. Sci. (RIMS), Kyoto, 2013, 191-212.

[24] Kapaev A.A., Asymptotic behavior of the solutions of the Painlevé equation of the first kind, Differential Equations 24 (1988), 1107-1115.

[25] Kawai T., Takei Y., WKB analysis of Painlevé transcendents with a large parameter. I, Adv. Math. 118 (1996), 1-33.

[26] Kawai T., Takei Y., Algebraic analysis of singular perturbation theory, Translations of Mathematical Monographs, Vol. 227, Amer. Math. Soc., Providence, RI, 2005.

[27] Kawakami H., Nakamura A., Sakai H., Degeneration scheme of 4-dimensional Painlevé-type equations, arXiv:1209.3836.

[28] Kontsevich M., Intersection theory on the moduli space of curves and the matrix Airy function, Comm. Math. Phys. 147 (1992), 1-23.

[29] Mulase M., Sułkowski P., Spectral curves and the Schrödinger equations for the Eynard-Orantin recursion, arXiv:1210.3006.

[30] Nakamura A., Autonomous limit of 4-dimensional Painlevé-type equations and degeneration of curves of genus two, arXiv:1505.00885.

[31] Norbury P., Quantum curves and topological recursion, arXiv:1502.04394.

[32] Okamoto K., Polynomial Hamiltonians associated with Painlevé equations. I, Proc. Japan Acad. Ser. A Math. Sci. 56 (1980), 264-268.

[33] Olshanetsky M.A., Painlevé type equations and Hitchin systems, in Integrability: the Seiberg-Witten and Whitham Equations (Edinburgh, 1998), Gordon and Breach, Amsterdam, 2000, 153-174, math-ph/9901019.

[34] Painlevé P., Sur les équations différentielles du second ordre et d'ordre supérieur dont l'intégrale générale est uniforme, Acta Math. 25 (1902), 1-85.

[35] Takasaki K., Spectral curves and Whitham equations in isomonodromic problems of Schlesinger type, Asian J. Math. 2 (1998), 1049-1078, solv-int/9704004.

[36] Takei Y., An explicit description of the connection formula for the first Painlevé equation, in Toward the Exact WKB Analysis of Differential Equations, Linear or Non-Linear (Kyoto, 1998), Kyoto University Press, Kyoto, 2000, 271-296. 